\title{
A business imperative to act sustainably
}

\section{Thea Sherer}

Businesses must understand and act to reduce their environmental footprint. Springer Nature has been working to address the impacts of its publishing operations since 2017, lowering office-based carbon emissions and improving sustainable practices.

As one of the world's largest publishers of academic research, educational content and science journalism, Springer Nature is a trusted source of information on environmental issues such as climate change and sustainability. However, the company's responsibilities extend beyond disseminating evidence-based research to academics, the general public and policy makers. We must also address environmental issues directly; employees, regulators, investors and customers all expect and demand efforts be taken to reduce the environmental footprint of our operations, and thereby contribute to environmental action under the auspices of the Paris Agreement and the UN Sustainable Development Goals.

As part of a wider Responsible Business strategy, Springer Nature has taken several steps to minimise its environmental impacts. The carbon footprint of our offices has been measured and reported since 2017, allowing strategic areas of improvement to be identified. An $18 \%$ reduction in net carbon emissions was achieved between 2017 and 2018, largely due to a trebling of the proportion of renewable energy used in the offices. While weather conditions played a part in reduced energy consumption at some of the larger sites, energy-efficiency measures also made an impact. For example, in one large warehouse in Argentina, the installation of LED lighting reduced energy consumption by $13 \%$.

As a global company with dispersed teams and customers, air travel represents a large proportion - around 50\% - of Springer Nature's carbon footprint. During 2019, Springer Nature's Environment Committee encouraged colleagues to travel less and to choose greener travel options such as travelling by train. We further developed a carbon-balancing pilot, offsetting approximately one-third of the year's business-related flight emissions. The offsets will fund reforestation and forestry protection projects in Nicaragua and Tanzania. Such initiatives aim at greatly contributing to Springer Nature's aim of becoming carbon neutral in the medium term.

After analysing other areas of considerable environmental impact, the paper used
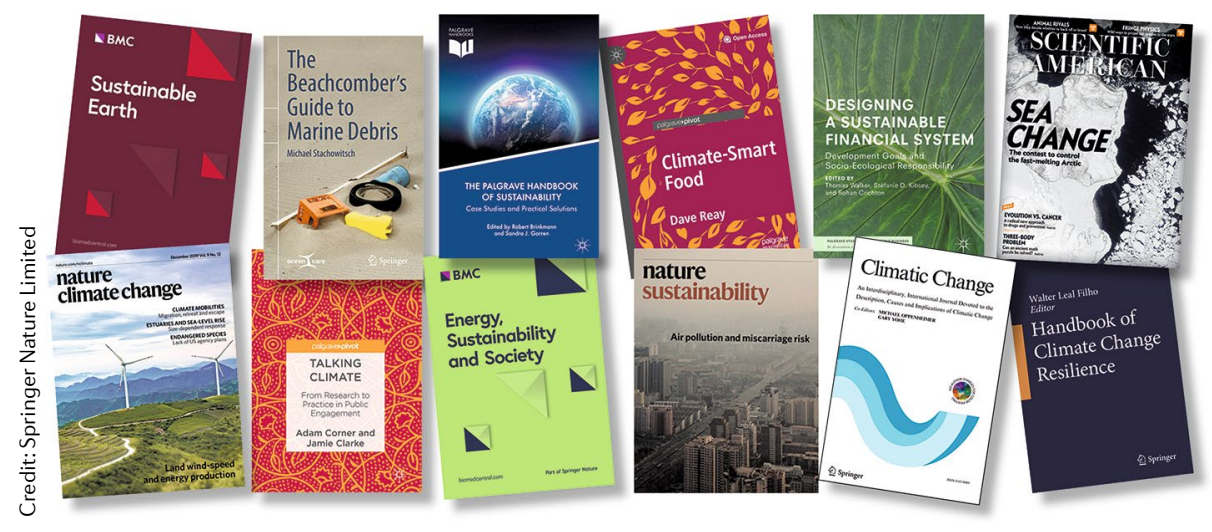

for printed products (text books, journals and magazines) was also targeted as a priority for improvement. A strengthened policy was developed and shared with suppliers to ensure that the paper supply chain only uses timber from legal, known and responsible sources that do not threaten important carbon sinks or other highly biodiverse ecosystems.

A network of green office ambassadors has also been established. This group of more than 100 global colleagues volunteer to help identify smaller changes that can add up to make a difference: recycling rates, energy efficiency and resource use. One initiative in London, for example, reduced the number of single-use coffee cups being used by 86,000 in the first quarter of 2018 alone. Other initiatives include expanding and better communicating local recycling options and encouraging more people to cycle to work.

Springer Nature is committed to reducing its operational environmental impacts and has made progress over the past 2 years, but recognizes there is more to do. Through its Sustainable Development Goals publishing programme, the company is also seeking to create new partnerships to have a greater impact on climate action and other global challenges. Collective efforts from across the business community are necessary to tackle the many environmental issues facing the planet, so we encourage businesses to start reporting and to start taking action.

Thea Sherer Springer Nature, London, UK. e-mail: thea.sherer@springernature.com https://doi.org/10.1038/s43017-019-0009-2

Competing interests

Thea Sherer is Director of Group Communications at Springer Nature and oversees the company's responsible business strategy. 